\title{
Impactos ambientais da fertilização orgânica em sistemas agropecuários na região sul-brasileira.
}

\author{
Paulo Hentz' ; Nathália Leal de Carvalho² \\ 1,2 Alunos do Programa de Pós-Graduação em Agronomia da Universidade de Passo Fundo, Passo Fundo, RS, Brasil
}

\section{Resumo}

A aplicação de fertilizantes orgânicos permite maior concentração do nutriente P nas camadas superficiais do solo, este deslocamento às camadas mais profundas ocorre de maneira lenta, principalmente, em solos argilosos, com maior quantidade de sítios de ligação. A aplicação de fertilizante orgânico na forma líquida quando injetado em sulcos e aplicado em faixas reduzem a emissão de $\mathrm{N}-\mathrm{NH}_{3}$, porém elevam a

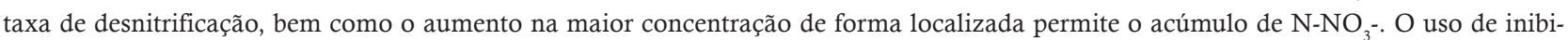
dores de nitrificação permitem menores perdas de $\mathrm{N}$ quando se aplica fertilizantes orgânicos, em razão da desconexão entre a produção de $\mathrm{N}-\mathrm{NO}_{3}$ - e o pico de metabolismo do $\mathrm{C}$ do esterco prontamente metabolizado. Doses crescentes de fertilizantes orgânicos promove maior atividade microbiana, a qual é muitas vezes mensurada pela maior emissão de $\mathrm{CO}_{2}$, sendo que a maior taxa de liberação ocorre logo após a aplicação no solo.

Palavras-chave: lixiviação, inibidores de nitrificação

\begin{abstract}
The application of organic fertilizers allows greater concentration of $\mathrm{P}$ in the nutrient topsoil, however, this shift to the deeper layers occurs slowly, especially on clay soil with higher binding sites. The application of organic fertilizer in liquid form when injected into grooves and applied in bands reduce the emission of $\mathrm{NH}_{3}-\mathrm{N}$, but raise the rate of denitrification, as well as the largest increase in concentration of localized form allows the accumulation of $\mathrm{N}_{-} \mathrm{NO}_{3}$ - before to be absorbed by plants. The application of organic fertilizers on straw hinders tillage fertilizer-soil contact and access to microorganisms for subsequent immobilization that may favor volatilization losses and lower utilization by plants. Increasing doses of organic fertilizer promotes greater microbial activity, which is often measured by higher $\mathrm{CO}_{2}$ emissions, with the highest rate of release occurs soon after soil application.
\end{abstract}

Keywords: leaching, nitrification, inhibitors. 


\section{INTRODUÇÃO}

A bovinocultura, suinocultura e avicultura apresentam grande destaque dentre as atividades agropecuárias desenvolvidas no Brasil, seguindo a demanda interna e externa de incremento por proteína animal, onde a perspectiva de crescimento anual para cada atividade é de 2,15, 2,0 e 3,64\% ao ano (PALHARES, 2012). Essas atividades são responsáveis por geração de renda e de emprego, principalmente nas pequenas e médias propriedades do sul do país. Portanto, a atividade para reciclagem destes resíduos da pecuária torna-se fundamental em termos de valor agronômico e impactos ambientais (LOVANH et al., 2010).

$\mathrm{Na}$ região Sul do Brasil, as atividades pecuárias ocorrem principalmente em pequenas propriedades, projetando concentrações produzidos por área e que em muitos casos são dificultadas de serem utilizados na agricultura por razões como topografia, falta de área agrícola da própria propriedade, falta de critério técnico para recomendação de adubação e quanto a sua forma de aplicação, sistemas produtivos pouco eficientes quanto a produção de grãos ou biomassa seca vegetal no caso de pastagens, falta de manejo de conservação do solo, aspectos que se forem levados em consideração promovem o uso incorreto de fertilizantes orgânicos com impactos negativos diretos na qualidade ambiental.

Em razão do pequeno espaço para aplicação dos dejetos, a maioria dos produtores aplica elevadas taxas de esterco nas mesmas áreas, como forma de eliminação do excesso de produção de esterco, sem levar em consideração as necessidades nutricionais das plantas cultivadas elevando os riscos de possível contaminação do solo e da água.

São poucos os trabalhos, que relatam o efeito dos fertilizantes orgânicos em relação ao impacto de nutrientes no solo e na água, sendo que a grande maioria deste está relacionado a suinocultura, havendo pouca referência à pecuária de bovinocultura de leite e avicultura, as quais contribuem de forma semelhante, ou até mesmo superiores em determinadas regiões quanto ao impacto no ambiente. Desta forma, a maior parte das referências será denotada a pecuária suinícola.

Nos Estados Unidos, França e Canadá, a criação de suínos é de forma intensiva, a aplicação de dejetos de suínos causou a contaminação do solo e da água, o que provocou mudanças na legislação ambiental como meio de proteger o ambiente (JONGBLOED et al., 1999). A União Européia (UE), estabelece que a quantidade máxima de nitrogênio $(\mathrm{N})$ oriundo de dejetos que pode ser aplicada sobre uma zona vulnerável é limitada a $170 \mathrm{~kg} \mathrm{ha}^{-1} \mathrm{ano}^{-1}$ (BERGSTRÖM \& KIRCHMANN, 2006).

Na França, Holanda e Dinamarca, grandes produtores de suínos, têm modificado suas legislações ambientais, visando aumentar a proteção ambiental e impedir o avanço da contaminação do solo e da água (JONGBLOED et al., 1999).

No Brasil, em Santa Catarina, o licenciamento ambiental para implantação ou expansão da suinocultura em propriedade só ocorre se o produtor comprovar possuir área de terra suficiente para o descarte dos dejetos produzidos, sendo permitido o máximo de $50 \mathrm{~m}^{3} \mathrm{ha}^{-1}$ ano $^{-1}$ de dejeto líquido de suínos (FATMA, 2004). No Rio Grande do Sul, o órgão de licenciamento não estabelece dose máxima de dejetos líquidos de suínos (DLS) a ser aplicada ao solo, mas enfatiza que seu uso como fertilizante deve ter como referencial a quantidade de nutrientes requeridos pelas culturas, baseado em análise de solo (FEPAM, 2004).

Devido à predominância do sistema de plantio direto em mais de $80 \%$ do total de terras cultiváveis no Sul do Brasil (AMADO et al., 2006), os DLS são aplicados predominantemente na superfície do solo, e aplicações contínuas ocasionam aumentos na quantidade de nutrientes, como o $\mathrm{N}$, fósforo (P) e potássio $(\mathrm{K})$, na superfície do solo, excedendo exigências nutricionais das culturas (ADELI et al., 2003) e aumentando o potencial de transferência por escoamento superficial (CERETTA et al, 2010).

Cerca de $60 \%$ do $\mathrm{N}$ contido no esterco é encontrado na forma de amônio, o qual é rapidamente transformado em nitrato no solo (PAYET et al., 2009). Como geralmente a aplicação do dejeto ocorre antes da semeadura, e se ocorrer intensa precipitação poderá haver movimentação vertical de $\mathrm{NO}_{3}{ }^{-}$no solo, principalmente, durante os estádios iniciais de desenvolvimento da cultura, quando a demanda de N é pequena (McCRACKEN et al., 1994).

A maior parte do $\mathrm{P}$ de dejetos de suínos está na forma inorgânica (CASSOL et al., 2001), sendo a principal forma de acumulação no solo (HOODA et al., 2001). A aplicação de dejetos a longo prazo promove a diminuição de adsorção de $\mathrm{P}$ e pode conferir maior escoamento superficial (BERWANGER et al., 2008). 
Elevadas concentrações de $\mathrm{P}, \mathrm{N}$ e $\mathrm{C}$ na água, causam danos ambientais e à saúde humana e animal, estando o $\mathrm{P}$ e o $\mathrm{N}$ associados a eutrofização, sendo $\mathrm{P}$ considerado o elemento limitante, pois $\mathrm{N}$ atmosférico pode entrar por fixação simbiótica por algas, como as Diazotrophicas sp (DANIEL et al., 1998).

\section{FÓSFORO E NITROGÊNIO}

Os motivos que levantados pelo excesso de $\mathrm{P}$ no solo em razão do grande volume de dejetos e a disponibilidade de pequenas áreas para o descarte, bem como agricultores fazem aplicações sucessivas de dejetos na mesma área não respeitando as instruções técnicas para a aplicação de doses adequadas. Não levando em consideração que a quantidade aplicada deve ser determinada de acordo com a concentração de nutrientes (N, P e K) e pelo índice de eficiência, o que indica o total de nutrientes contidos no esterco que podem ser transformados a partir da forma orgânica para a forma mineral depois da aplicação no solo (CQFS RS/SC, 2004).

Assim, aplicações sucessivas ou em excesso de $\mathrm{P}$ ao longo do tempo numa mesma área, promove aumento nas concentrações no solo acima da demanda das culturas, ocasionando seu acúmulo na superfície (GIROTTO et al., 2010), que pode favorecer possíveis escoamentos superficiais.

A aplicação de fertilizantes orgânicos ao longo do tempo promove alterações nas frações de $\mathrm{P}$ no solo, assim como a saturação dos sítios de adsorção por esse elemento (BERWANGER et al., 2008) especialmente na forma inorgânica resultando numa menor capacidade de adsorção de $\mathrm{P}$ no perfil. Isto ocorre de tal modo, que a energia de ligação do P com os colóides do solo diminua, aumentando sua disponibilidade às plantas e potencializando as transferências de fósforo por escoamento superficial e percolação (BASSO et al., 2005; GIROTTO et al., 2010), causando problemas ambientais.

$\mathrm{O}$ aporte contínuo permite concentrações superiores as que a cultura consegue exportar no sistema, permite que os sítios de fixação sejam preenchidos e, há o deslocamento da frente de adsorção para colóides do solo a profundidades maiores, sendo esta percolação favorecida pela maior velocidade de difusão, além da forma de deslocamento por lixiviação através de canais naturais e bioporos na presença da água móvel do solo, pela preservação das características físicas do solo para estes sistemas de produção, portanto a existência de canais contínuos resultantes da decomposição de raízes e atividade biológica, permite a migração de $\mathrm{P}$ na forma orgânica.

$\mathrm{O}$ acúmulo do $\mathrm{P}$ no solo está relacionado com a quantidade do elemento que é adicionada ao solo, do tempo de aplicação, da quantidade aplicada, da composição do dejeto, do tipo de solo, e exportação deste elemento pelas culturas no sistema de produção (CERETTA et al., 2003). Espera-se que ao longo dos anos, aplicações contínuas de $\mathrm{P}$ na superfície do solo pode levar à ocupação da superfície de adsorção, reduzindo a energia de adsorção de $\mathrm{P}$ e, aumento da sua dessorção e disponibilidade para as plantas (BOLLAND et al., 1996).

Com a aplicação de fertilizantes orgânicos em sistema de produção plantio direto (SPD) temse verificado o acúmulo de $\mathrm{P}$, o que permite maior eficiência de aproveitamento deste nutriente, e faz com que não ocorra ganhos significativos de produtivo com adubações fosfatadas pesadas (RESENDE et al., 2012).

CERETTA et al. (2010) em experimento a campo com DLS aplicaram 508; 1016 e $2032 \mathrm{~kg}$

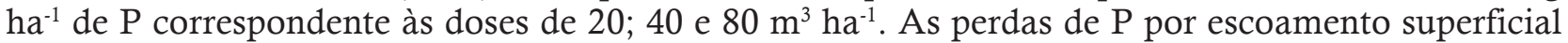
foram, 32; 51 e $71 \mathrm{~kg} \mathrm{ha}^{-1}$, e correspondem as perdas de 6,4;10,2 e 14,4 $\mathrm{kg} \mathrm{ha}^{-1} \mathrm{ano}^{-1}$, para a testemunha a perda média foi de $2,4 \mathrm{~kg} \mathrm{ha}^{-1} \mathrm{ano}^{-1}$.

As perdas de $\mathrm{P}$ por escoamento superficial não estão relacionados com o volume de escoamento, mas como observado para o nitrato, as maiores concentrações de P permitiram um maior escoamento nas primeiras coletas após a aplicação dos dejetos (CERETTA et al., 2005).

A maior parte do P adicionado ficou acumulado na camada superficial. Enquanto que na camada de 0-10 cm, onde não foi aplicado esterco, o P no solo foi $12,8 \mathrm{mgkg}^{-1}$ e quando foram, aplicadas doses de 20; 40 e $80 \mathrm{~m}^{3} \mathrm{ha}^{-1}$ de esterco aplicados, os níveis de P foram 122,0; 275,9 e 753,9 mg $\mathrm{kg}^{-1}$ (CERETTA et al., 2010).

Isto é justificado pelo fato que o $\mathrm{P}$ é adsorvido na maior parte por sítios ávidos, mas como o esterco tem altos valores de $\mathrm{P}$, pode ocorrer a saturação destes locais e se torna $\mathrm{P}$ adsorvido em fra- 
ções com energia de ligação inferior (BARROW et al., 1998), que pode ser biodisponível e facilmente deslocado pelo escoamento superficial, logo após a aplicação de DLS (KLEINMAN et al., 2009).

As concentrações de $\mathrm{P}$ no escoamento superficial eram maiores que $0,15 \mathrm{mg} \mathrm{L}^{-1}$ nas primeiras amostras, atingindo a concentração máxima permitida para a água classe 3 , a qual pode ser utilizada para consumo humano após tratamento adequado (CONAMA, 2005). Isto indica que a aplicação sucessiva de esterco aumentou as perdas de $\mathrm{P}$ por escoamento superficial, provocando a contaminação das fontes de água para consumo humano, bem como o aumento do risco de eutrofização dos ambientes aquáticos (SMITH et al., 2007).

Aplicações anuais de esterco em altas doses, em SPD visando o suprimento integral das necessidades de $\mathrm{N}$ da cultura do milho $\left(120 \mathrm{kgha}^{-1} \mathrm{de} \mathrm{N}\right)$ podem, em médio e longo prazos resultar em acúmulo de P na camada superficial do solo (SCHERER \& NESI, 2009). Da mesma forma a adubação orgânica, aumenta a disponibilidade de $\mathrm{P}$ na camada de $10-20 \mathrm{~cm}$, indicando certa migração do nutriente em profundidade (EGHBALL et al., 2000), em culturas anuais e pastagem nativa (CERETTA et al., 2003).

$\mathrm{O}$ deslocamento do $\mathrm{P}$ no solo pode ter ocorrido pela preservação das características físicas do solo no SPD e pela existência de canais contínuos resultantes da decomposição de raízes e atividade biológica, permitindo a migração de $\mathrm{P}$ na forma orgânica, onde as camadas mais profundas ficaram com teor de $3,0 \mathrm{mg} \mathrm{dm}^{-3}$, não diferindo do tratamento sem adubação, valor considerado baixo (CFQS RS/SC, 2004). Comportamento semelhante foi observado no perfil de solos intensivamente adubados com DLS (SCHERER \& NESI, 2009) e esterco de bovinos (SILVA et al., 2007).

Verifica-se que, mesmo com altos teores de $\mathrm{P}$ nas camadas superficiais, o deslocamento do elemento para camadas profundas é relativamente lento, principalmente em solos argilosos, como é o caso dos Latossolos da Região Oeste de Santa Catarina (SCHERER \& NESI, 2009).

$\mathrm{O}$ aporte anual de $\mathrm{N}$ e $\mathrm{P}$ em áreas com culturas mercantis pode ser elevado a dose média de esterco de $16 \mathrm{mgha}^{-1}$ ( $15 \%$ umidade) com teores de $\mathrm{N}$ e $\mathrm{P}$ de 9 e $3 \mathrm{gkg}^{-1}$. Com isso, haveria excesso para essa de 62 e $37 \mathrm{kgha}^{-1}$ de $\mathrm{N}$ e P. A visão de adubação do sistema e não de uma safra/cultura, tem se mostrado um aspecto preponderante para um bom manejo nutricional das lavouras, rotação de culturas e as produtividades obtidas tem implicações nos estoques e na ciclagem de nutrientes no ambiente de produção (SILVA et al., 2007).

$\mathrm{O} \mathrm{N}$ é o macronutriente exigido em maior quantidade pela maior parte das culturas agrícolas, o fator que dificulta a recomendação de adubação de forma eficiente é razão de sua dinâmica no solo, que envolve reações como: imobilização/mineralização; nitrificação/desnitrificação; lixiviação/volatilização entre outros, os quais são provenientes da interação entre microrganismos e colóides do solo e mediados por fatores climáticos de difícil previsão. Outra dificuldade está relacionada a ele não estar presente na análise básica de rotina de solos em razão das dificuldades metodológicas em avaliar sua disponibilidade (CANTARELLA \& DUARTE, 2004).

$\mathrm{O}$, além da eutrofização, está associado a problemas com saúde humana, como a síndrome do bebê-azul ou meta-hemoglobinemia, alteração na oxigenação do sangue causada pelo nitrato $\left(\mathrm{N}-\mathrm{NO}_{3}\right.$ ). O nitrato também tem sido associado a doenças cancerígenas (SMITH et al., 2001). O íon amônio $\left(\mathrm{N}_{-} \mathrm{NH}_{4}^{+}\right)$, que é convertido em amônia $\left(\mathrm{N}_{-} \mathrm{NH}_{3}^{+}\right)$e vice-versa, em função do $\mathrm{pH}$, pode alterar a vida

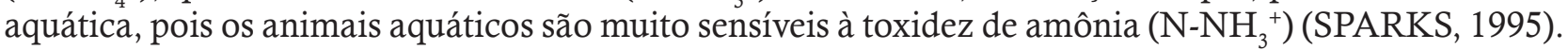

Quanto ao escoamento superficial, o $\mathrm{N}$ divide-se em duas formas, solúvel e particulada. A forma solúvel é constituída pelo $\mathrm{N}_{-} \mathrm{NO}_{3}{ }^{-} \mathrm{e} \mathrm{N}-\mathrm{NH}_{4}{ }^{+}$, sendo imediatamente disponível à vida aquática. Já a forma particulada corresponde à fração que se encontra adsorvida às partículas sólidas do solo ou, como constituinte das partículas orgânicas do solo (SHARPLEY et al., 1987).

Para o $\mathrm{N}$ na forma de $\mathrm{N}_{-} \mathrm{NO}_{3}^{-}$, a lixiviação é o principal processo envolvido no transporte do solo, devido à baixa capacidade de retenção, na maioria dos solos (EGHBALL \& GILLEY, 1999).

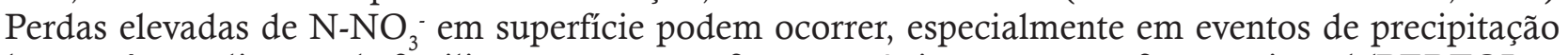
logo após a aplicação de fertilizantes, tanto na forma orgânica quanto na forma mineral (BERTOL et al., 2005). Embora as perdas de $\mathrm{N}_{-} \mathrm{NO}_{3}{ }^{-}$via superfície geralmente sejam pequenas, outras formas de $\mathrm{N}$, como $\mathrm{N}_{-} \mathrm{NH}_{4}^{+}$e N particulado, podem representar perdas significativas via escoamento superficial. Nos DLS, além do $\mathrm{N}$ orgânico, é encontrado o $\mathrm{N}^{-} \mathrm{NH}_{4}^{+}$, apesar de haver grande variação em função de fatores como a idade dos animais, tipo de alimentação e sistema de manejo (HOODA et al., 2001).

A expectativa com relação ao incremento de doses de dejeto animal é de que a concentração e a quantidade perdida de $\mathrm{C}$ e $\mathrm{N}$ via escoamento superficial aumente. Aplicações sucessivas de adubos 
elevam as concentrações dos nutrientes no solo, aumentando, o potencial de transporte de nutrientes do solo para a água (SCHERER \& AITA, 1996; BERTOL et al., 2005), fato que se agrava no sistema plantio direto, devido à não incorporação dos fertilizantes (SCHICK et al., 2000). Sabe-se que SPD é muito eficiente no controle das perdas de solo, mas, também, que os solos apresentam capacidade máxima de infiltração e que, mesmo em SPD e após esse limite o escoamento estará, associado ao impacto ambiental (MELLO et al., 2003).

Experimentos com chuvas após a aplicação de DLS, têm mostrado maiores perdas de solo, água e nutrientes, possivelmente em função do selamento superficial causado pelo material orgânico (BERTOL et al., 2005; MORI et al., 2009). No entanto, experimentos de longa duração indicam menores perdas de solo, água e nutrientes, em razão dos efeitos benéficos do dejeto na qualidade física do solo, diminuindo o escoamento superficial (SMITH et al., 2001).

A aplicação de até $120 \mathrm{~m}^{3} \mathrm{ha}^{-1} \mathrm{ano}^{-1}$ de dejeto líquido de bovino em solo manejado sob plantio direto reduziu as perdas de $\mathrm{C}$ orgânico e $\mathrm{N}$ por escoamento superficial, com conseqüências positivas para a qualidade da água em corpos hídricos. A aplicação de doses maiores pode aumentar a concentração dos referidos elementos e, diminuir a qualidade da água (SILVEIRA et al., 2011).

\section{INJEÇÃO DE ESTERCOS}

No Brasil a adoção de uma nova tecnologia tem despertado o interesse pelos pesquisadores que é a utilização de sistemas de injeção de estercos líquidos. A incorporação de esterco em lavoura logo após a aplicação é conhecido por reduzir grandemente as perdas de $\mathrm{N}$ devido à volatilização da amônia $\left({\left.\mathrm{N}-\mathrm{NH}_{3}\right)}\right.$ (WEBB \& MISSELBROOK, 2004). O esterco permanece na superfície do solo em SPD ou produção de gramíneas forrageiras.

Do ponto de vista agronômico, menores perdas de $\mathrm{N}-\mathrm{NH}_{3}$, permite a conservação de $\mathrm{N}$ disponível para as plantas. Reduzir as emissões de $\mathrm{N}-\mathrm{NH}_{3}$ pode também beneficiar a qualidade do ar e da água. A preocupação com a qualidade do ar que podem ser tratadas através da redução das emissões de $\mathrm{N}-\mathrm{NH}_{3}$ é a formação de material particulado atmosférico e efeitos associados sobre a saúde humana (PINDER et al., 2007). A diminuição das emissões de $\mathrm{N}^{-\mathrm{NH}_{3}}$ podem reduzir a quantidade de $\mathrm{N}$ atmosférico que será redepositado em ecossistemas naturais, onde contribui para acidificação do solo, e contribui para a eutrofização. Estima-se que cerca de $6 \%$ das entradas de $\mathrm{N}$ para a bacia são derivadas da deposição atmosférica de origem agrícola (BOBBINK et al., 2010).

Aplicações de esterco em subsuperfície são teoricamente susceptíveis a maiores perdas por desnitrificação devido à disponibilidade de $\mathrm{C}$ que impulsiona a atividade microbiológica com $\mathrm{N}_{-} \mathrm{NO}_{3}^{-}$, resultante da nitrificação do $\mathrm{N}_{-} \mathrm{NH}_{4}{ }^{+}$(WULF et al., 2002). Aplicações subsuperficiais podem aumentar

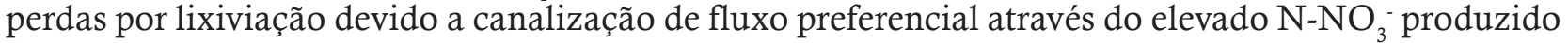
a partir da nitrificação e mineralização do N orgânico do esterco (SHIPITALO \& GIBBS, 2000).

Potencialmente estas maiores perdas por desnitrificação e lixiviação podem reduzir a volatilização de $\mathrm{N}-\mathrm{NH}_{3}$. A nitrificação e desnitrificação também tem sua importância devido à sua contribuição para as emissões de $\mathrm{N}_{2} \mathrm{O}$, que é um gás de efeito estufa (GEE), que é cerca de 300 vezes mais eficaz do que o $\mathrm{CO}_{2}$ na absorção de radiação na atmosfera (IPCC, 2007).

RODHE et al. (2006) relataram 275\% maior emissão de $\mathrm{N}_{2} \mathrm{O}$ com esterco bovino injetado em comparação com a aplicação de esterco em faixas em superfície, no entanto, as emissões de $\mathrm{N}_{2} \mathrm{O}$ globais em seu estudo foram baixas (1,1 e $0,3 \%$ do $\mathrm{N}$ total do esterco para injeção e aplicado em faixas).

A injeção de produto cofermentado (70\% esterco de gado leiteiro, 30\% de resíduos orgânicos domésticos) resultou em duas a três vezes maior emissão de $\mathrm{N}_{2} \mathrm{O}$, em comparação com a aplicação por placa de respingo, na lavoura e pastagens (WULF et al., 2002). Embora as emissões de $\mathrm{N}_{2} \mathrm{O}$ não foram medidos separadamente por (THOMPSON et al., 2002), um aumento das emissões de $\mathrm{N}_{2} \mathrm{O}$ foram associados com a maior atividade de desnitrificação total observada com injeção de esterco. Outros estudos mostram que a injeção de esterco não afetou as emissões de $\mathrm{N}_{2} \mathrm{O}$ (VALLEJO et al., 2005; VELTHOF et al., 2009). 


\section{EMISSÕES DE AMÔNIA}

Os estudos apontam grande variações na redução de $\mathrm{N}_{-} \mathrm{NH}_{3}$ entre 40 a 90\%. As maiores reduções nas emissões têm sido observadas com injetores e fechamento do sulco, quando contrastados com injetores que não fecham o sulco (WEBB et al. 2009).

As reduções substanciais em perdas de $\mathrm{N}-\mathrm{NH}_{3}$ podem ser esperados com a aplicação de esterco em subsuperfície em comparação com a aplicação em superfície. Estas reduções são alcançadas através da limitação da área da superfície do esterco que está exposta ao ar e através do aumento da imobilização de $\mathrm{N}-\mathrm{NH}_{4}{ }^{+}$devido ao maior contato do chorume com o solo.

Fatores como o modelo do aplicador, umidade do solo, teor de matéria seca do esterco e taxa de aplicação, podem influenciar a emissão de $\mathrm{N}_{-} \mathrm{NH}_{3}$ por controlar a colocação e distribuição do esterco aplicado no solo. Em geral, as emissões de $\mathrm{N}^{-\mathrm{NH}_{3}}$ devem aumentar em proporção com a quantidade de área superficial do solo coberta pelo esterco.

HANSEN et al. (2003) encontraram relação linear entre o volume do compartimento criado pelo aplicador e o potencial para reduzir as emissões de $\mathrm{N}_{-} \mathrm{NH}_{3}$, indicando que é necessária fechar suficientemente o sulco para assegurar que o esterco não permaneça sobre a superfície do solo.

Isso indica que taxas de aplicação não podem exceder o volume dos sulcos disponíveis para a eficaz contenção de dejeto aplicado. Aplicadores em sulcos abertos ou fechados podem ter performance semelhante em pastagens (RODHE et al., 2006), mas a aplicação com sulco aberto nem sempre pode reduzir as emissões de $\mathrm{N}-\mathrm{NH}_{3}$ em terras cultiváveis (WEBB et al., 2009). A umidade do solo e teor de matéria seca do esterco influencia a capacidade dos aplicadores em subsuperfície para reduzir as emissões de $\mathrm{N}-\mathrm{NH}_{3}$. Em solos úmidos, o fechamento do sulco diminui a infiltração do esterco (SOMMER \& ERSBOLL, 1994). O aumento do conteúdo de matéria seca também diminui a taxa de infiltração do esterco no solo (PETERSEN et al., 2003).

As reduções de emissões de $\mathrm{N}-\mathrm{NH}_{3}$ podem ser alcançadas com aplicações em faixas na superfície próximo a cultura. MISSELBROOK et al. (2002) constataram que as emissões de $\mathrm{N}^{-\mathrm{NH}_{3}}$ em sete locais na Inglaterra com aplicação em faixas e próximo a cultura foram de 57 e $26 \%$ mais baixo do que com aplicação a lanço.

A aplicação de esterco de gado de leite em faixas injetado reduziu as emisões de $\mathrm{N}_{-} \mathrm{NH}_{3} \mathrm{em}$ cerca de 50\% em comparação com aplicação a lanço (BITTMAN et al., 2005).

O efeito de aplicações subsuperficais podem variar de acordo com o tipo de injetor, profundidade de injeção e umidade do solo. A injeção pode reduzir a perda de $\mathrm{N}^{-\mathrm{NH}_{3}}$ de 40 a $90 \%$ em comparação com a aplicação de DLS em superfície não incorporado. Além disso, a redução na emissão de N-NH com injeção é imediata, ao contrário da incorporação pelo preparo do solo, o que requer uma operação separada. Em torno de $50 \%$ das emissões de $\mathrm{N}_{-} \mathrm{NH}_{3}$ pode ser esperado dentro de $24 \mathrm{~h}$ na aplicação a lanço, assim a incorporação por uma operação de cultivo separada deve ser feito rapidamente após a aplicação para alcançar significativa redução (THOMPSON et al., de 2002).

\section{PERDAS DE NITROGÊNIO DEVIDO A DESNITRIFICAÇÃO}

Aplicações de esterco em subsuperfície pode levar a maior desnitrificação e subsequente perdas de $\mathrm{N}$ como gás $\mathrm{N}_{2}$ ou $\mathrm{N}_{2} \mathrm{O}$. Grandes adições de compostos de $\mathrm{C}$ facilmente metabolizados pode resultar em altas taxas de atividade microbiana dentro do sulco de injeção, esgotando o oxigênio e criando condições anaeróbias necessárias para a desnitrificação (FLESSA \& BEESE, 2000; WULF et al., 2002). O potencial de perdas devido à desnitrificação também pode aumentar se o $\mathrm{N}$ conservado pela redução da volatilização de $\mathrm{N}-\mathrm{NH}_{3}$ se acumular como $\mathrm{N}_{-} \mathrm{NO}_{3}^{-}$antes de ser utilizada pelas plantas.

As perdas de $\mathrm{N}$ total por desnitrificação foram 76 e $293 \%$ maiores quando o esterco de foi injetado no sulco, em comparação a aplicação a lanço. Embora a injeção permita a conservação de 50 a $75 \mathrm{~kg} \mathrm{Nha}^{-1}$ em razão da redução pela emissão de $\mathrm{N}^{-\mathrm{NH}_{3}}$ (THOMPSON et al., 2002).

A injeção de esterco resultou em aumento de $62 \%$ na perda por desnitrificação $\left(\mathrm{N}_{2} \mathrm{O}+\mathrm{N}_{2}\right)$, apesar de reduzir as emissões de $\mathrm{N}_{-} \mathrm{NH}_{3}$ em $91 \%$ em comparação com a aplicação em superfície (DOSCH \& GUTSER, 1996). Neste caso, a redução das perdas devido à volatilização de $\mathrm{N}^{-\mathrm{NH}_{3}}$ foi de $20 \mathrm{~kg} \mathrm{Nha}^{-1}$, mas houve adicional de perda por desnitrificação de $3 \mathrm{~kg}$ de $\mathrm{Nha}^{-1}$. Perdas por desni- 
trificação variando de 0,3 a $8,1 \mathrm{~kg} \mathrm{Nha}^{-1}$ com injeção superficial do esterco de gado, mas perceberam pouca diferença entre a injeção e aplicação superficial (MISSELBROOK et al., 1996).

\section{EFEITOS SOBRE A LIXIVIAÇÃO}

As perdas de $\mathrm{N}$ por lixiviação são influenciadas pela precipitação, propriedades do solo, topografia, e manejo do solo. No entanto, a colocação de esterco em faixas concentradas abaixo do solo pode aumentar a lixiviação de $\mathrm{N}$ se as faixas de esterco interceptar as vias de fluxo preferenciais.

A injeção no Outono de lodo de esgoto digerido para solo argiloso na Inglaterra resultou em 32 a $500 \%$ a mais na lixiviação de $\mathrm{N}_{-} \mathrm{NO}_{3}^{-}$a uma profundidade de 1 metro do que quando o mesmo material foi aplicado na superfície. Em adição para o grande potencial de lixiviação do solo areno argiloso, elevadas aplicações de $\mathrm{N}-\mathrm{NH}_{4}{ }^{+}$com lodo digerido $\left(60-300 \mathrm{kgha}^{-1}\right)$, e a subsequente formação de $\mathrm{N}_{-} \mathrm{NO}_{3}^{-}$, pode ter exacerbadas perdas por lixiviação (SHEPHERD, 1996).

BALL-COELHO et al. (2006) verificaram aumento da lixiviação de $\mathrm{N}_{-} \mathrm{NO}_{3}$ - quando se aplica DLS injetado em altas taxas, mas não observaram impacto do método de aplicação quando adições de $\mathrm{N}$ do esterco não excederam os requerimentos da cultura. A lixiviação de $\mathrm{N}-\mathrm{NO}_{3}$ - para drenos $(\sim 90 \mathrm{~cm}$ de profundidade) não foi afetada pela injeção do chorume de suínos em trincheira rasa (WESLIEN et al., 1998). As perdas de $\mathrm{N}^{-\mathrm{NO}_{3}}$ por lixiviação a profundidade de $90 \mathrm{~cm}$ eram baixos porque as taxas aplicadas não eram excessivas (THOMPSON et al., 2002).

$\mathrm{O}$ balanço do $\mathrm{N}$ para o chorume mostra que as perdas de $\mathrm{N}-\mathrm{NH}_{3}$ foi de $1 \%$, em comparação com $31 \%$ do $\mathrm{N}$ total (NT) perdido na aplicação superficial. Porém, as perdas gasosas pela desnitrificação eram $21 \%$ do NT com injeção, em comparação com $12 \%$ com a aplicação superficial. As perdas totais de gases a partir da aplicação superficial foram $43 v s 22 \%$ do NT da aplicação por injeção. As menores perdas gasosas e maior conservação de $\mathrm{N}$ disponível para as plantas com injeção também foram expressos em maior resposta pelas culturas pelo $\mathrm{N}$, com recuperação estimada em $33 \%$ do $\mathrm{N}$ via injeção do chorume, em comparação com $20 \%$ das aplicações em superfície (THOMPSON et al., 2002).

As perdas por desnitrificação foram muito menores após a aplicação na primavera (7\% do NT para injeção e $2 \%$ para aplicação superficial). Estes resultados foram atribuídos às baixas condições de umidade do solo e maior utilização pela cultura, com recuperação pela cultura de $36 \%$ do NT para injeção e $26 \%$ para aplicações em superfície. A adição de nitrapirina ao chorume injetado aumentou a recuperação de $\mathrm{N}$ pela cultura em $42 \%$ do NT, mas produziu perdas similares de $\mathrm{N}_{-} \mathrm{NH}_{3}(1 \%)$ e as perdas por desnitrificação (5\%) comparado com o chorume não injetado (THOMPSON et al., 2002).

\section{IMPLICAÇÕES PARA EMISSÕES DE GASES DE EFEITO ESTUFA (GEE)}

Os métodos de aplicação de dejetos em solos agrícolas necessitam de maiores pesquisas, pois tem influência direta na emissão de $\mathrm{CO}_{2}$, volatilização de $\mathrm{N}-\mathrm{NH}_{3}, \mathrm{~N}_{2} \mathrm{O}$ para a atmosfera.

A dinâmica do $\mathrm{N}$ no sistema solo-panta em SPD apresenta velocidades de reações diferentes quando comparado ao sistema conservacional, sendo que, quando a aplicação de dejetos é realizada em superfície sobre a palha de culturas anteriores dificulta o contato solo-resíduo e, o acesso aos microorganismos as fontes de energia e carbono, reduzindo a demanda microbiana por $\mathrm{N}$.

$\mathrm{O}$ efeito do $\mathrm{N}$ do dejetos sobre a decomposição da palhada do trigo e cevada e mostrou que a quantidade de $\mathrm{CO}_{2}$ liberada em solos que receberam simultaneamente dejetos de suínos e palha foi significativamente maior do que a soma de $\mathrm{CO}_{2}$ emitida pelos tratamentos com apenas dejetos líquidos de suínos ou apenas palha, indicando interação aditiva quando dejetos e palha foram incorporados juntos. A falta de qualquer efeito dos DLS na mineralização do $C$ da palhada em condições de campo para o fato da ocorrência de chuvas logo após a aplicação dos dejetos, o cátion amônio $\left(\mathrm{N}^{-} \mathrm{NH}_{4}^{+}\right)$dos dejetos provavelmente foi lixiviado abaixo da camada de palha em decomposição (CHANTIGNY et al., 2002).

As maiores emissões de $\mathrm{CO}_{2}$ ocorreram em tratamentos onde a palha foi incorporada no solo, ou seja, sozinha ou com dejetos de suínos e, quando dejetos e palhada foram aplicados na superfície do solo. A mineralização do $\mathrm{C}$ dos DLS foi muito rápida nos primeiros dias. A diferença na minera- 
lização do $C$ em dejetos de suínos esta relacionada a sua localização e ocorre imediatamente após a adição de dejetos, quando a taxa de liberação de $\mathrm{CO}_{2}$ foi máxima (AITA et al., 2012).

$\mathrm{O}$ pico de liberação de $\mathrm{CO}_{2}$ ocorre logo após a aplicação DLS em a campo e em condições controladas. Isto foi atribuído à rápida e intensa mineralização dos ácidos graxos voláteis presentes nos dejetos e também à presença de carbonatos em DLS, acumulados durante o armazenamento anaeróbio e que pode ser liberado no solo, sem o consumo de $\mathrm{O}_{2}$ (CHANTIGNY et al., 2002; ROCHETTE et al., 2004).

Este resultado pode ser explicado pela biodegradação dos compostos mais instáveis durante o armazenamento anaeróbio do esterco de suínos. As proporções entre esses compostos mais facilmente degradáveis e compostos mais recalcitrantes remanescentes após o armazenamento de DLS pode determinar as diferenças observadas na taxa de mineralização do $C$ deste material orgânico (MARCATO et al., 2009).

Cerda de 59\% do C orgânico adicionado com dejetos de suínos evoluiu como $\mathrm{CO}_{2}$, independente da forma de colocação dos dejetos no solo. A qualidade e composição da dieta animal e da duração da armazenagem dos dejetos antes da aplicação no solo são as variáveis que podem explicar estas discrepâncias na literatura sobre a mineralização do C de dejetos de suínos (GIACOMINI et al., 2007).

Quando DLS são aplicados sobre a palhada de trigo o crescimento de microorganismos é favorecido (AITA et al., 2012).

Quando a palha de trigo e DLS foram adicionados juntos e incorporada no solo, o contato resíduo-solo-cultura e disponibilidade de $\mathrm{N}$ foram favoráveis para a decomposição de palha, uma vez que esta prática favorece a colonização microbiana da palhada (POTTHOFF et al., 2005) e impede a perda de $\mathrm{N}$ dos dejetos de suínos pela volatilização de $\mathrm{N}_{-} \mathrm{NH}_{3}$ (ROCHETTE et al., 2004).

$\mathrm{O}$ uso de tecnologias para a injeção de dejetos de suínos no solo sob resíduos de culturas com elevada relação $\mathrm{C} / \mathrm{N}$ pode ser a estratégia mais desejável para favorecer a retenção do $\mathrm{C}$ da palha $\mathrm{e}$ $\mathrm{N}$ dos dejetos de suínos no solo, quando os dois materiais orgânicos são utilizados em conjunto em sistemas agrícolas (AITA et al., 2012).

É necessária a análise econômica, ambiental da agricultura sobre o potencial das maiores emissões de $\mathrm{N}_{2} \mathrm{O}$ pela injeção de esterco para avaliar o custo ambiental total e potencial responsabilidade econômica para os agricultores. As quantidades de $\mathrm{N}$ perdidas como $\mathrm{N}_{2} \mathrm{O}$ são uma pequena parte do NT aplicado e não constituem uma grande perda econômica para o agricultor, a compreensão do custo total de maiores emissões de $\mathrm{N}_{2} \mathrm{O}$ reside principalmente no potencial impacto sobre os impactos globais de GEE e do potencial para a mudança climática global.

Painel Intergovernamental sobre Mudanças Climáticas (IPCC, 2007) estima que, em média, $1,25 \%$ do nitrogênio total do fertilizante ou esterco aplicado é emitido como $\mathrm{N}_{2} \mathrm{O}$.

Portanto, o esterco de gado de leite com $4 \mathrm{~g}$ de $\mathrm{NTL}^{-1}$, aplicado em superfície a uma taxa de 75.000 $\mathrm{Lha}^{-1}$ pode produzir emissões de $3,75 \mathrm{kgha}^{-1} \mathrm{~N}_{2} \mathrm{O}$. Com relatos sobre emissões de $\mathrm{N}_{2} \mathrm{O}$ de até $300 \%$, podemos antecipar que a injeção de esterco levaria a emissões de até $12 \mathrm{~kg} \mathrm{~N}_{2} \mathrm{Oha}^{-1}(\sim 19 \mathrm{~kg}$ $\mathrm{N}_{2} \mathrm{Oha}^{-1}$ ). $\mathrm{O}$ valor de fertilizantes de $\mathrm{N}$ perdido como $\mathrm{N}_{2} \mathrm{O}$ ainda seria provavelmente menos do que $\mathrm{O}$ valor do $\mathrm{N}$ conservado pela redução das emissões de $\mathrm{N}^{2} \mathrm{NH}_{3}$.

O uso de inibidores de nitrificação como a dicianodiamida (DCD), com esterco tem potencial para diminuir as perdas de desnitrificação e as emissões de $\mathrm{N}_{2} \mathrm{O}$, causando uma desconexão entre a produção de $\mathrm{N}_{-} \mathrm{NO}_{3}^{-}$e o pico de metabolismo do $\mathrm{C}$ do esterco prontamente metabolizado (COMFORT et al.,1990).

A adição da DCD pode eliminar perdas adicionais por desnitrificação resultantes da injeção de esterco bovino. Adicionando DCD com esterco resultou em $30 \%$ maior rendimento de forragem e 156\% maior recuperação do N aparente (MISSELBROOK et al., 1996).

A adição de DCD em dejetos de suínos efetivamente inibiu a nitrificação durante 20 a 30 dias e as emissões de $\mathrm{N}_{2} \mathrm{O}$ reduzidas em aproximadamente $46 \%$ para injeção e aplicação superficial. Quando DMPP foi aplicado a uma taxa de $2 \mathrm{kgha}^{-1} \mathrm{com}$ chorume bovino, houve redução de $32 \%$ na emissão de $\mathrm{N}_{2} \mathrm{O}$ em comparação com e sem injeção de inibidor de nitrificação. Adicionalmente, o uso de $\mathrm{N}^{15}$ marcado do esterco mostrou que as emissões e a atividade do inibidor foram localizadas dentro da sulco de injeção (VALLEJO et al., 2005). 


\section{CONSIDERAÇÕES FINAIS}

A aplicação de fertilizantes na forma orgânica quanto ao impacto ambiental é em grande parte provocada pela falta de critérios técnicos para recomendação de adubação ao sistema, associada a longo tempo de aplicação e altas concentrações utilizadas de uma única vez o que pode gerar saturação dos sítios de adsorção por esse elemento, resultando numa menor capacidade de adsorção de $\mathrm{P}$ no perfil, bem como diminui a energia de ligação que o $\mathrm{P}$ faz com os colóides do solo, o que aumenta a dessorção, disponibilidade às plantas e potencializa as transferências de fósforo por escoamento superficial e percolação.

A aplicação de fertilizantes orgânicos permite a construção da fertilidade do solo para $\mathrm{P}$ e torna possível maior eficiência de aproveitamento e proporciona a planta explorar todo o seu potencial genético produtivo. Desta forma a prática de aplicação de fertilizantes orgânicos na condição de acúmulo de $\mathrm{P}$ pode vir a ser anti-econômica, restringindo o impacto ambiental.

A aplicação de fertilizante orgânico na forma líquida injetado em sulcos, bem como a aplicação em faixas permitem reduzir a emissão de N-NH3 no sistema de produção, no entanto, estas práticas elevam a taxa de desnitrificação em razão de conduzir a ambientes anaeróbios, seja pelo maior excesso de umidade e/ou menor aeração, bem como o aumento na maior concentração de forma localizada

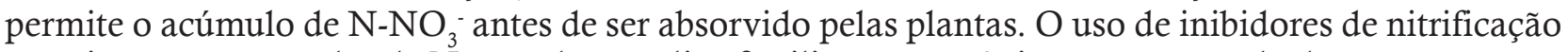
permitem menor perdas de $\mathrm{N}$ quando se aplica fertilizantes orgânicos, em razão da desconexão entre a produção de $\mathrm{N}_{-} \mathrm{NO}_{3}^{-}$e o pico de metabolismo do $\mathrm{C}$ do esterco prontamente metabolizado.

A injeção permite maior lixiviação de nitrogênio em razão da maior concentração localizada deste nutriente no solo, principalmente se as faixas de esterco interceptar as vias de fluxo preferenciais de água no solo (canais naturais e bioporos), e em alguns casos as perdas de $\mathrm{N}$ por volatilização chegam a alcançar o dobro do valor quando aplicado na superfície do solo.

Doses crescentes de fertilizantes orgânicos promove maior atividade microbiana, a qual é muitas vezes mensurada pela maior emissão de $\mathrm{CO}_{2}$, sendo que a maior taxa de liberação ocorre após a aplicação no solo, sendo este resultado explicado pela biodegradação dos compostos orgânicos de baixo peso molecular.

\section{REFERÊNCIAS}

ADELI, A. et al. Swine effluent irrigation rate and timing effects on bermudagrass growth, nitrogen and phosphorus utilization and residual soil nitrogen. Journal of Environmental Quality, 32, p.681-686, 2003.

AITA, C. et al. Nitrificação do nitrogênio amoniacal de dejetos líquidos de suínos em solo sob sistema plantio direto. Pesquisa Agropecuária Brasileira, 42, p.95-102, 2007.

AITA, C. et al. Impact on $\mathrm{C}$ and $\mathrm{N}$ dynamics of simultaneous application of pig slurry and wheat straw, as affected by their initial locations in soil. Biology Fertility Soils, 48, 633-642, 2012. DOI 10.1007/s00374-0110658-x

AMADO, T.J.C. Potential of carbon accumulation in no till soils with intensive use and cover crops in southern Brazil. Journal Environmental Quality, 35, p.1599-1607, 2006.

ANDRASKI, T.W. et al. Manure history and long-term tillage effects on soil properties and phosphorus losses in runoff. Journal Environmental Quality, 32, p.1782-1789, 2003.

BALL-COELHO, B.R. et al. Nitrogen recovery and partitioning with diff erent rates and methods

of sidedressed manure. Soil Science Society of AmericaJournal, 70:464-473, 2006.

BARROW, N.J. et al. Effect of previous additions of superphosphate on sorption of phosphate. Australian Journal Soil Research, v.36, 359-372, 1998. 
BASSO, C.J. et al. Dejeto líquido de suínos: II-Perdas de nitrogênio e fósforo por percolação no solo sob plantio direto. Ciência Rural, v.35, n.6, p.1305-1312, 2005.

BERWANGER, A.L.et al. Alterações no teor de fósforo no solo com aplicação de dejetos líquidos de suínos. Revista Brasileira de Ciência do Solo, 32, 2525-2532, 2008.

BERGSTRÖM, L.; KIRCHMANN, H. Leaching and crop uptake of nitrogen and phosphorus from pig slurry as affected by different application rates. Journal Environmental Quality, 35, 1803-1811, 2006.

BERTOL, I. et al. Effect of a chiseling and rainfall erosivity on some parameters of water erosion in management systems of a typical Hapludox. Revista Brasileira de Ciência do Solo, 32, 747-757, 2008.

BITTMAN, S. et al. Surface-banding liquid manure over aeration slots: A new low-disturbance method for reducing ammonia emissions and improving yield of perennial grasses. Agronomy Journal, 97,1304-1313, 2005.

BOBBINK, R. et al. Global assessment of nitrogen deposition eff ects on terrestrial plant diversity: A synthesis. Journal of Applied Ecology, 20, 30-59, 2010.

BOLLAND, M.D.A. et al. Comparison of seven phosphorus sorption indices. Australian Journal Soil Research, 34,81-89, 1996.

CASSOL, P.C. et al. Frações de fósforo em estrumes e sua eficiência como adubo fosfatado. Revista Brasileira de Ciência Solo, 25, 635-644, 2001.

CERETTA, C.A. et al. Frações de fósforo no solo após sucessivas aplicações de dejetos de suínos em plantio direto. Pesquisa Agropecuária Brasileira. 45, 593-602, 2010.

CERETTA, C.A. et al. Características químicas de solo sob aplicação de esterco líquido de suínos em pastagem natural. Pesquisa Agropecuária Brasileira, 38:729-735, 2003.

CHANTIGNY, M.H. et al. Fate of carbon and nitrogen from animal manure and crop residues in wet and cold soils. Soil Biologycal Biochemistry, 34, 509-517, 2002.

COMFORT, S.D. et al. Nitrous oxide production from injected liquid dairy manure. Soil Science Society of America Journal, 54, 421-427, 1990.

COMISSÃO DE QUÍMICA E FERTILIDADE DO SOLO - CQFS RS/SC. Manual de adubação e calagem para os estados do Rio Grande do Sul e Santa Catarina. Porto Alegre: SBCS/NRS/UFRGS, 2004. 400p.

CONAMA-Conselho Nacional do Meio Ambiente. Resolução CONAMA N`357. CONAMA, Brasília, 2005 Dispnível em: from:http://www.crq4.org.br/downloads/ resolucao357.pdf. Acesso em 23 abril 2013.

DAVEREDE, I.C. et al. Phosphorus runoff: Effect of tillage and soil phosphorus levels. Journal Environmental Quality, 32, 1436-1444, 2003.

DANIEL, T.C. et al. Agricultural phosphorus and Eutrophication: A Symposium Overview. Journal Environmental Quality, 27, 251-257, 1998.

DINNES, D.L. et al. Nitrogen management strategies to reduce nitrate leaching in tiledrained midwestern soils. Agronomy Journal, 94, 153-171, 2002.

DOSCH, P.; GUTSER, R. Reducing N losses (NH3, N2O, N2) and immobilization from slurry through optimized application techniques. Fertility Research, 43, 165-171, 1996. 
EGHBALL, B.; GILLEY, J.E. Phosphorus and nitrogen in runoff following beef cattle manure or compost application. Journal Environmental Quality, 28, 1201-1210, 1999.

EGHBALL, B. et al. Phosphorus movement and absorption in a soil receiving longterm manure and fertilizer application. Journal of Environmental Quality, v.25, p.1339-1343, 2000.

FEPAM-Fundação Estadual de Proteção Ambiental Henrique Luis Roessler. Critérios técnicos para o licenciamento ambiental de novos empreendimentos destinados à suinocultura. FEPAM, Porto Alegre, 2004.

FLESSA, H.; BEESE, F. Laboratory estimates of trace gas emissions following surface application and injection of cattle slurry. Journal Environmental Quality, 267, 117-127, 2000.

GATIBONI, L. C. et al. Formas de fósforo no solo após sucessivas adições de dejeto líquido de suínos em pastagem natural. Revista Brasileira de Ciência do Solo, v. 32, n. 4, p. 1753-1761, 2008.

GIACOMINI, S.J. et al. Simulating the effects of N availability, straw particle size and location in soil on C and N mineralization. Plant Soil, 301,289-301, 2007.

GIROTTO, E. et al. Formas de perdas de cobre e fósforo em água de escoamento superficial e percolação em solo sob aplicações sucessivas de dejeto líquido de suínos. Ciência Rural, v.40, n.9, p.1860-1866, 2010.

GORDON, R. et al. Soil aeration for dairy manure spreading on forage: Effect on ammonia volatilization and yield. Canadian Journal of Soil Science, 80, 319-326, 2000.

GROOT, J.C.J. et al. Interpretation of results from on-farm experiments: Manure-nitrogen recovery on grassland as aff ected by manure quality and application technique. 1. An agronomic analysis. Wageningen Journal of Life Science, 54, 235-254, 2007.

HANSEN, M. et al. Reduction of ammonia emission by shallow slurry injection: Injection effi ciency and additional energy demand. Journal Environmental Quality, 32, 1099-1104, 2003.

HART, M.R. et al. Phosphorus runoff from agricultural land and direct fertilizer effects: a review. Journal Environmental Quality, 33, 1954-1972, 2004.

HOODA, P.S. et al. Manuring and fertilization effects on phosphorus accumulation in soils and potential environmental implications. Advances in Environmental Research, 5, 13-21, 2001.

IPCC. Synthesis report. Intergovernmental Panel on Climate Change, 2007. Disponível em: www.ipcc.ch/ publications. Acesso em 18 maio 2013.

JONGBLOED, A.W. et al. Environmental and legislative aspects of pig production in the Netherlands, France and Denmark. Livestock Production Science, 58, 243-249, 1999.

KAY, P. et al. A review of the efficacy of contemporary agricultural stewardship measures for ameliorating water pollution problems of key concern to the UK water industry. Agricultural Systems, 99, 67-75, 2009.

KLEINMAN, P.J.A. et al. Application of manure to no-till soils: phosphorus losses by sub-surface an surface pathways. Nutrients Cycling Agroecosystems, 84, 215-227, 2009.

LOVANH, N. et al. Determination of ammonia and greenhouse gas emissions from land application of swine slurry: a comparison of three application methods. Bioresource Technology, 101, 1662-1667, 2010.

MARCATO, C.E. et al. Impact of anaerobic digestion on organic matter quality in pig slurry. International Biodeterioration and Biodegradation, 63, 260-266, 2009. 
McCRACKEN, D.V. et al. Nitrate leaching as influenced by cover cropping and nitrogen sourse. Soil Science Society of America Journal, v.58, n.5, p.1476-1483, 1994.

MELLO, E.L. et al. Perdas de solo e água em diferentes sistemas de manejo de um Nitossolo Háplico submetido à chuva simulada. Revista Brasileira de Ciência do Solo, 27, 901-909, 2003.

MISSELBROOK, T.H. et al. Surface application and shallow injection of cattle slurry on grassland: Nitrogen losses, herbage yield and nitrogen recoveries. Grass Forage Science, 51, 270-277, 2002.

MORI, H.F. et al. Perda de água, solo e fósforo com aplicação de dejeto líquido bovino em latossolo sob plantio direto e com chuva simulada. Revista Brasileira de Ciência do Solo, 33, 89-198, 2009.

MYERS, T.L. Master's thesis. Pennsylvania State Univ. University Park, PA. 2010.

PAERL, H.W. Coastal eutrophication and harmful algal blooms: Importance of atmospheric deposition and groundwater as new nitrogen and other nutrient sources. Limnology \& Oceanography, 42, 1154-1165, 1997.

PALHARES, J.C. Impacto ambiental das produções pecuárias. Congresso brasileiro de animais sustentável. Anais...EMBRAPA: Brasília.2012. 17p.

PAYET, N. et al. Modelling the fate of nitrogen following pig slurry application on a tropical cropped acid soil on the Island of Réunion (France). Agricultural Ecosystems Environmental, 134, 218-233, 2009.

PETERSEN, S.O. et al. Redistribution of slurry components as infl uenced by injection method, soil, and slurry properties. Journal Environmental Quality, 32, 2399-2409, 2003.

PINDER, R.W. et al. Ammonia emission controls as a cost-eff ective strategy for reducing atmospheric particulate matter in the eastern United States. Environmental Science Technology, 41, 380-386, 2007.

POTTHOFF, M. et al. Dynamics of maize (Zea mays L.) leaf straw mineralization as affected by the presence of soil and the availability of nitrogen. Soil Biological Biochemistry, 37, 1259-1266, 2005.

RANDALL, G.W.; MULLA, D.J. Nitrate nitrogen in surface waters as influenced by climatic conditions and agricultural practices. Journal Environmental Quality, 30, 337-344, 2001.

ROCHETTE, P. et al. Ammonia volatilization and soil nitrogen dynamics following fall application of pig slurry on canola crop residues. Canadian Journal of Soil Science, 82, 515-523, 2004.

RODHE, L. et al. Nitrous oxide, methane and ammonia emissions following slurry spreading on grassland. Soil Use Management, 22, 229-237, 2006.

SOMMER, S.G.; SHERLOCK, R.R. pH and buffer component dynamics in the surface layers of animal slurries. Journal Agricultural Science, 127, 109-116, 1996.

SOMMER, S.G.; ERSBOLL, A.K. Soil tillage eff ects on ammonia volatilization from surface-applied or injected animal slurry. Journal Environmental Quality, 23, 493-498, 1994.

SPARKS, D.L. Environmental Soil Chemistry. San Diego, Academic Press, 1995. 267p.

SHARPLEY, A.N. et al. Environmental impact of agricultural nitrogen and phosphorus use. Journal Agricultural Food Chemistry, 35, 812-817, 1987.

SCHERER, E.E.; AITA, C. Avaliação da qualidade do dejeto líquido de suíno da região oeste catarinense para fins de utilização como fertilizante. Florianópolis, EPAGRI, 1996. (Boletim Técnico, 79). 
SCHERER, E.E.; NESI, C.N. Características químicas de um Latossolo sob diferentes sistemas de preparo e adubação orgânica. Bragantia, v.68, n.3, p.715-721, 2009.

SCHICK, J. et al. Erosão hídrica em Cambissolo Húmico alumínico submetido a diferentes sistemas de preparo e cultivo do solo. II - Perdas de nutrientes e carbono orgânico. Revista Brasileira de Ciência do Solo, 24, 437-447, 2000.

SHEPHERD, M.A. Factors aff ecting nitrate leaching from sewage sludges applied to a sandy soil in arable agriculture. Agricultural Ecosystems Environmental, 58, 171-185, 1996.

SHIPITALO, M.J.; GIBBS, F. Potential of earthworm burrows to transmit injected animal wastes to tile drains. Soil Science Society of America Journal, 64, 2103-2109, 2000.

SILVA, T.O. et al. Adubação orgânica da batata com esterco e/ou Crotalaria juncea. I - Produtividade vegetal e estoque de nutrientes no solo em longo prazo. Revista Brasileira de Ciência do Solo, v.31, p.39-49, 2007.

SMITH, D.R. et al. Applications as impacted by time to first runoff event. Environmental Pollution, 147, 131-137, 2007.

SURESH, A. et al. Prediction of the nutrients value and biochemical characteristics of swine slurry by measurement of EC-electrical conductivity. Bioresource Technology, 100, 4683-4689, 2009.

THOMPSON, R.B. et al. Management factors aff ecting ammonia volatilization from land-applied cattle slurry in the Mid-Atlantic USA. Journal Environmental Quality, 31, 1329-1338, 2002.

VALLEJO, A. et al. Comparison of $\mathrm{N}$ losses (NO3-, N2O, NO) from surface applied, injected or amended (DCD) pig slurry of an irrigated soil in a Mediterranean climate. Plant Soil, 272, 313-325, 2005.

VELTHOF, G.L. et al. Integrated assessment of nitrogen losses from agriculture in EU-27 using MITERRA-EUROPE. Journal Environmental Quality, 38, 402-417, 2009.

WEBB, J et al. Review of published studies estimating the abatement effi cacy of reduced-emission slurry spreading techniques. In M. Sutton, S. Reis, and S.M.H. Baker (ed.) Atmospheric ammonia. Springer Science + Business Media B.V., Dordrecht, the Netherlands, 2009.

WESLIEN, P. et al. Nitrogen losses following application of pig slurry to arable land. Soil Use Management, $14,200-208,1998$. 